\title{
Identification of Nuclear Localization Signals of Drosophila G9a Histone H3 Methyltransferase
}

\author{
Yasuko Kato ${ }^{\dagger}$, Yuta Ushijima, and Masamitsu Yamaguchi* \\ Department of Applied Biology and Insect Biomedical Research Center, Kyoto Institute of Technology, \\ Matsugasaki, Sakyo-ku, Kyoto 606-8585, Japan
}

\begin{abstract}
G9a is one of the well-characterized histone methyltransferases. G9a regulates H3K9 mono- and dimethylation at euchromatic region and consequently plays important roles in euchromatic gene regulation. Mammalian G9a contains several distinct domains, such as GHD (G9a homology domain), ANK, preSET, SET and PostSET. These domains are highly conserved between mammals and Drosophila. Although mammalian G9a has nuclear localization signal (NLS) in its $\mathrm{N}$-terminal region, the amino acid sequences of this region are not conserved in Drosophila. Here we have examined the subcellular localization of a series of truncated forms of Drosophila G9a (dG9a). The identified region (aa337-aa470) responsible for nuclear localization of dG9a contains four short stretches of positively charged basic amino acids (NLS1, aa334-aa345; NLS2, aa366-aa378; NLS3, aa407-aa419; NLS4, aa461-aa472). Each of NLS1, NLS3 and NLS4 is sufficient for the nuclear localization when they are fused with the enhanced green fluorescent protein. These NLSs of dG9a are distinct from those of mammalian G9a in their positions and amino acid sequences.
\end{abstract}

Key words: dG9a/nuclear localization signal/Drosophila

\section{Introduction}

In eukaryotic cells, covalent histone modification is critical for chromatin structure and defines epigenetic regulation for transcription, mitosis and heterochromatin. The binding of HP1 to histone $\mathrm{H} 3$ lysine 9 (H3-K9) following H3-K9 methylation is a key for constitutive heterochromatin (Martin and Zhang, 2005).

In Drosophila, H3-K9 mono-, di- and trimethylation is enriched in pericentromeric heterochromatin including the fourth chromosome in the polytene chromosome (Schotta et al., 2002; Ebert et al., 2004). SU(VAR)3-9 was first identified H3-K9-specific hintone methyltransferase (HMTase) (Czermin et al., 2001). In Su(var)3-9 null mutants, H3-K9 di- and trimethylation disappear from the chromocenter of

\footnotetext{
*To whom correspondence should be addressed: Masamitsu Yamaguchi, Department of Applied Biology, Kyoto Institute of Technology, Matsugasaki, Sakyo-ku, Kyoto 606-8585, Japan.

Tel: +81-75-724-7781, Fax: +81-75-724-7799

E-mail: myamaguc@kit.ac.jp

†resent address: Yasuko Kato, Laboratory for Germline Development, RIKEN Center for Developmental Biology, 2-2-3 Minatojima-minamimachi, Chuo-ku, Kobe 650-0047, Japan.

Abbreviations: NLS, nuclear localization signal; EGFP, enhanced green fluorescent protein; PI, propidium iodide; GHD, G9a homology domain; aa, amino acid.
}

the salivary gland chromosome but not from the heterochromatic fourth chromosome (Ebert et al., 2004). Furthermore, other two HMTases in Drosophila have been identified. One of them, dSETDB1 is required for gene silencing of the fourth chromosome (Seum et al., 2007; Tzeng et al., 2007; Brower-Toland et al., 2009) and is critical during development (Seum et al., 2007; Tzeng et al., 2007). Another HMTase, Drosophila G9a (dG9a) appears to be a functional homologue of human G9a and GLP/Eu-HMTase1 (Mis et al., 2006; Stabell et al., 2006). dG9a appears to have H3-K9 methyltransferase activity both in vitro and in vivo, and functions as a dominant suppressor of position effect of variegation (Mis et al., 2006). Although a reduced reproductivity of dG9a mutant flies suggests the involvement of dG9a in germ cell development (Mis et al., 2006; Lee et $a l ., 2010$ ), the other study indicates that dG9a is not essential for fly viability and reproduction (Seum et al., 2007). Furthermore, dG9a associates with Drosophila Atrophin which is a nuclear receptor co-repressor and is thought to be recruited to target gene promoters to repress their transcription (Mang et al., 2008). dG9a is important for the recruitment of HP1 in the euchromatic region to repress transcription (Kato et al., 2008). Over-expression of dG9a in eye imaginal discs inhibited the differentiation of pupal ommatidial cells and resulted in abnormal eye morphology (rough 
eye phenotype) in the adults, although a methylase defective mutant did not demonstrate such effects, suggesting that HMTase activity of dG9a affects transcription of genes involved in pupal eye formation (Kato et al., 2008). In addition, genetic interaction between dG9a and some Polycomb group genes as well as little imaginal discs were observed (Kato et al., 2008).

In mammals, G9a is one of the well-characterized HMTases. G9a regulates $\mathrm{H} 3 \mathrm{~K} 9$ mono- and dimethylation at euchromatic region and consequently plays important roles in euchromatic gene regulation. A variety of putative G9atarget genes have been identified and interestingly more than $70 \%$ of these genes are late-replicating (Yokochi et al., 2009). In mammals, G9a interacts with closely related homolog of G9a, GLP/Eu-HMTase1 during gene repression (Tachibana et al., 2005) and more recently a functional cooperation between the four H3K9 HMTases such as G9a, GLP, SETDB1 and Suv39h1 has been also reported (Fritsch et al., 2010). In Drosophila, dG9a is only an orthologue of mammalian G9a and GLP. Mammalian G9a contains several distinct domains, such as GHD (G9a homology domain), ANK, preSET, SET and PostSET. These domains are highly conserved between mammals and Drosophila. Although mammalian G9a has nuclear localization signal (NLS) in its N-terminal region (Estève et al., 2005), the amino acid sequences of this region are not conserved in Drosophila.

Here we have used a series of truncated forms of dG9a and identified the functional domains for the nuclear localization. The identified nuclear localization signals of dG9a are distinct from those of mammalian G9a in its position and amino acid sequence.

\section{Materials and Methods}

\section{Plasmid construction}

FLAG-tagged various deleted dG9a mutants were constructed with the pUAST-FLAG vector. The pUAST-FLAG-dG9a (Kato et al., 2008) was used as a template for PCR. To construct a series of plasmids expressing HA-dG9a, various deletion cDNA fragments were amplified by PCR and inserted into pActSV-HA vector. For detailed analysis of the nuclear localization signal, oligonucleotides were synthesized to construct the plasmid pUAST-EGFPdG9aNLS1, pUAST-EGFP-dG9aNLS2, pUAST-EGFP-dG9aNLS3, pUAST-EGFP-dG9aNLS4. After annealing the following pairs of synthetic oligonucleotids, the double stranded ologonucleotides were cloned into the pUAST-EGFP vector (Sugano and Yamaguchi, 2008).

The oligonucleotide EGFP-dG9aNLS1, 5'-GATCCCTGCTCCGAAGCGAGGAAGGGGTCGAGCCAGGAAATAGC-3', and 5'-TCGAGCTATTTCCTGGCTCGACCCCTTCCTCGCTTCGGAGCAGG. The oligonucleotide EGFP-dG9aNLS2, 5'-GATCGGGGAGAAGAACCAGGACGCAAAAGGAAACTCCCGGATT-
AGC-3', and 5'-TCGAGCTAATCCGGGAGTTTCCTTTTGCGTCCTGGTTTCTTCTCCCC-3'. The oligonucleotide EGFPdG9aNLS3, 5'-GATCGTTAAGAGAATGCGACGTAGCGTTCGTTTAGGCAATCGATAGC-3', and 5'-TCGAGCTATCGATTGCCTAAACGAACGCTACGTCGCATTCTCTTAAC. The oligonucleotode EGFP-dG9aNLS4, 5'-GATCAAGACCCCACCCAAAAAGCGAGGGCGGAAGGCAAAATAGC-3', and 5'-TCGAGCTATTTTGCCTTCCGCCCTCGCTTTTTGGGTGGGGTCTT-3'.

To construct plasmids expressing HA-dG9a carrying internal deletions, pAct-HA-dG9 $\mathrm{a}_{\triangle 334-472}$, pAct-HA-dG9a $\mathrm{a}_{\triangle 834-926}$ and pActHA-dG9 $\mathrm{a}_{\triangle 334-472 / 834-926}$ were created by chimera PCR. The following primers were synthesized. 5'-dG9a(481), 5'-GCAACGACGTCGACAGAGAGAT; 3'-dG9a(999), 5'-TAATAGAGGACTCCTTTTGAGCCGATGCGGGGA; 5'-dG9a(1417), 5'-CTCGGCTACGCCCTGTGAATCTGAAACATCCT; 3'-dG9a(2499), 5'-CGACAGGAGTCTCCAAGTGTTAGATGCCTGGA; 5'dG9a(2779), 5'-CAATCTACGGACCTCCACGGTGAC; 3'dG9a(3164), 5'-GTCTCAAATGGGGCGCCCTATA. To obtain chimera PCR products, two rounds of PCR were performed. First PCR was performed with primers 5'-dG9a(481) and 3'-dG9a(999), primers 5'-dG9a(1417) and 3'-dG9a(4914), 5'-dG9a(481) and 3'-dG9a(2499), primers 5'-dG9a(2779) and 3'-dG9a(3164), 5'dG9a(481) and 3'-dG9a(2499), respectively. Second PCR was performed with primers 5'-dG9a(481) and 3'-dG9a(3164). To create pAct-HA-dG9 $\mathrm{a}_{\triangle 334-472 / 834-926}$, pAct-HA-dG9 $\mathrm{a}_{\triangle 334-472}$ was used as a template for PCR. The PCR fragments were digested with SalI/ SacII, and then inserted into SalI/SacII sites of pActSV-HA-dG9a plasmid.

\section{Fluorescent microscope analysis}

$2 \times 10^{5} \mathrm{Kc}$ cells grown on 12 -well dishes were deposited on polylysine treated coverslips. The cells were fixed with $4 \%$ paraformaldehyde in PBS for $30 \mathrm{~min}$, and permeabilized with $0.2 \%$ Triton $\mathrm{X}-100$ in PBS for $5 \mathrm{~min}$. After washing with PBS, the cells were treated with $1 \mathrm{mg} / \mathrm{ml} \mathrm{RNaseA}$ for $30 \mathrm{~min}$ at $37^{\circ} \mathrm{C}$ followed by washing with PBS. The samples were blocked with $1 \%$ skim milk in PBS for $10 \mathrm{~min}$, then were incubated for $1 \mathrm{~h}$ with mouse monoclonal $\alpha$-HA (InvivoGen, San Diego, CA, USA) at 1:500 dilution, mouse monoclonal $\alpha$-FLAG (M2, Sigma, St Louis, MO, USA) at $5 \mu \mathrm{g} / \mathrm{ml}$, or rabbit polyclonal $\alpha$-dG9a (Kato et al., 2008) following by washing a further three times with $0.1 \%$ NP40 in PBS. Cells were blocked again with $1 \%$ skim milk in PBS for $10 \mathrm{~min}$, then incubated for $30 \mathrm{~min}$ with Alexa Fluor 488-conjugated secondary antibody (Molecular Probes [Invitrogen], Carlsbad, CA, USA) at $1: 400$ dilution. After washing three times with $0.1 \%$ NP-40 in PBS, the samples were stained with $100 \mu \mathrm{g} / \mathrm{ml}$ propidium iodide for $5 \mathrm{~min}$. Coverslips were mounted in Fluoroguard Antifade Reagent (Bio-Rad, Hercules, CA, USA), and examined under a confocal laser scanning microscope (LSM510, Carl Zeis, Jena, Germany).

\section{Cell culture and transfection}

Kc cells derived Drosophila melanogaster embryos were grown at 
A

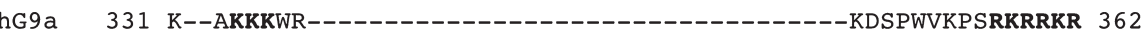

hGLP 393 KKFLKRKGK--------------------------------TDSPWIKPARKRRRR 416

dG9a 592 KRLFSKSQRDIENYGAAMLAKSKLPPCPDVEQFLNDIKASRINANRSPEERKLNKKQQR 651

$B$

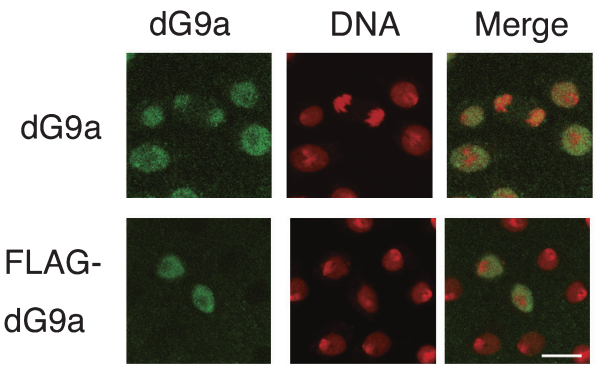

C

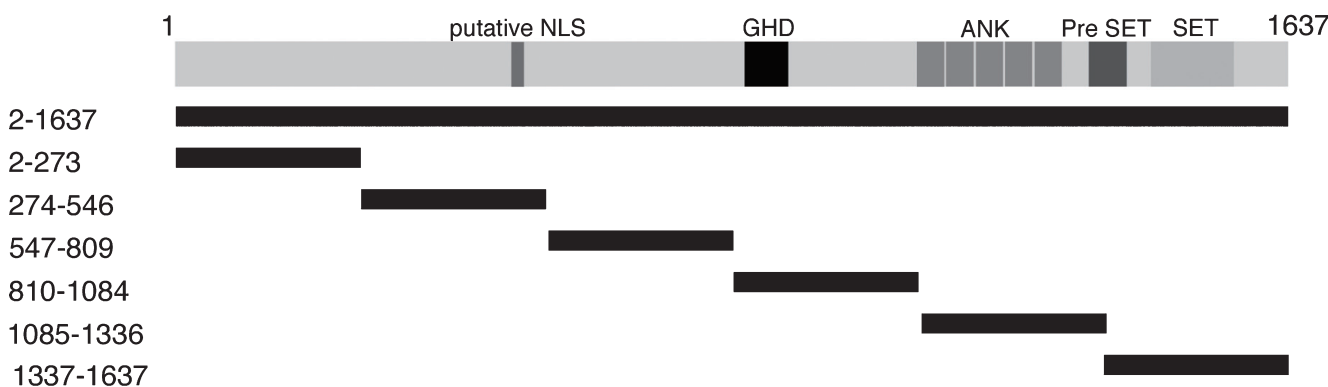

$1337-1637$

D

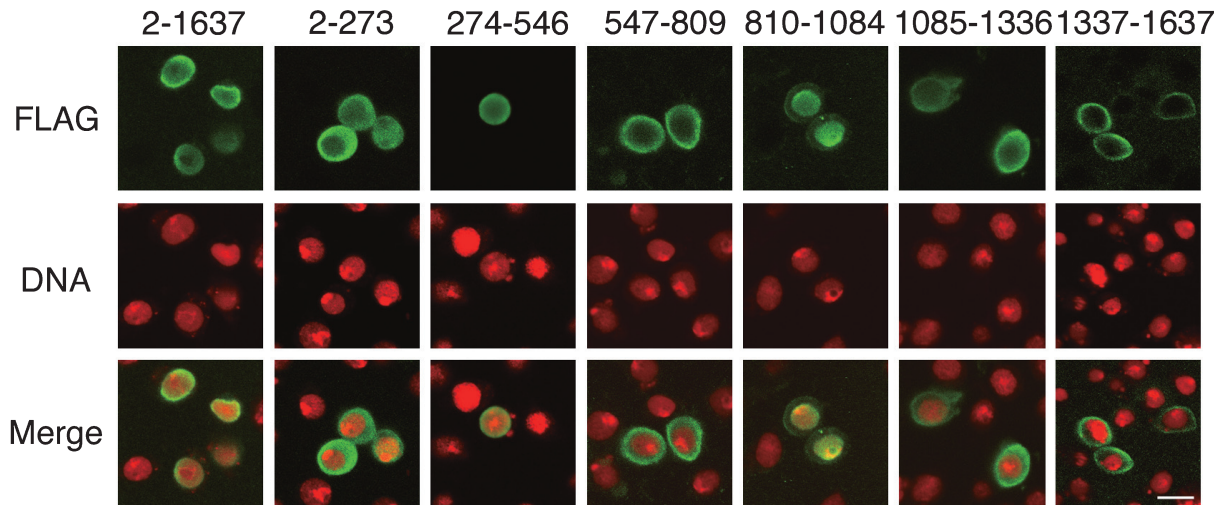

Fig. 1. Subcellular localization of endogenous dG9a and transfected full length and deletion mutants. (A) Sequence comparison of NLS region among hG9a, hGLP and dG9a using ClustalW (http://www.ebi.ac.uk/Tools/clustalw2/index.html). Conserved residues are marked by arrowheads. (B) Subceller localization of endogenous dG9a and FLAG-tagged dG9a in Kc cells. Kc cells were immunostained with $\alpha-\mathrm{dG} 9 \mathrm{a}$ antibody (upper panel, green). Kc cells transfected with FLAG-tagged dG9a and immunostained with $\alpha$-Flag antibody (lower panel, green). DNA was stained with PI (red). Scale bar, $5 \mu \mathrm{m}$. (C) A schematic diagram representing proteins of dG9a wild type and deletion mutants used to determine the subcellular localization of dG9a. GHD, ANK repeat, pre SET, and SET domains are indicated. NLS is a putative NLS searched with NLS database. (D) Confocal laser scanning microscopy of Kc cells transfected with FLAG-tagged proteins (green). DNA was stained with propidium iodide (PI, red). Scale bar, $5 \mu \mathrm{m}$. 

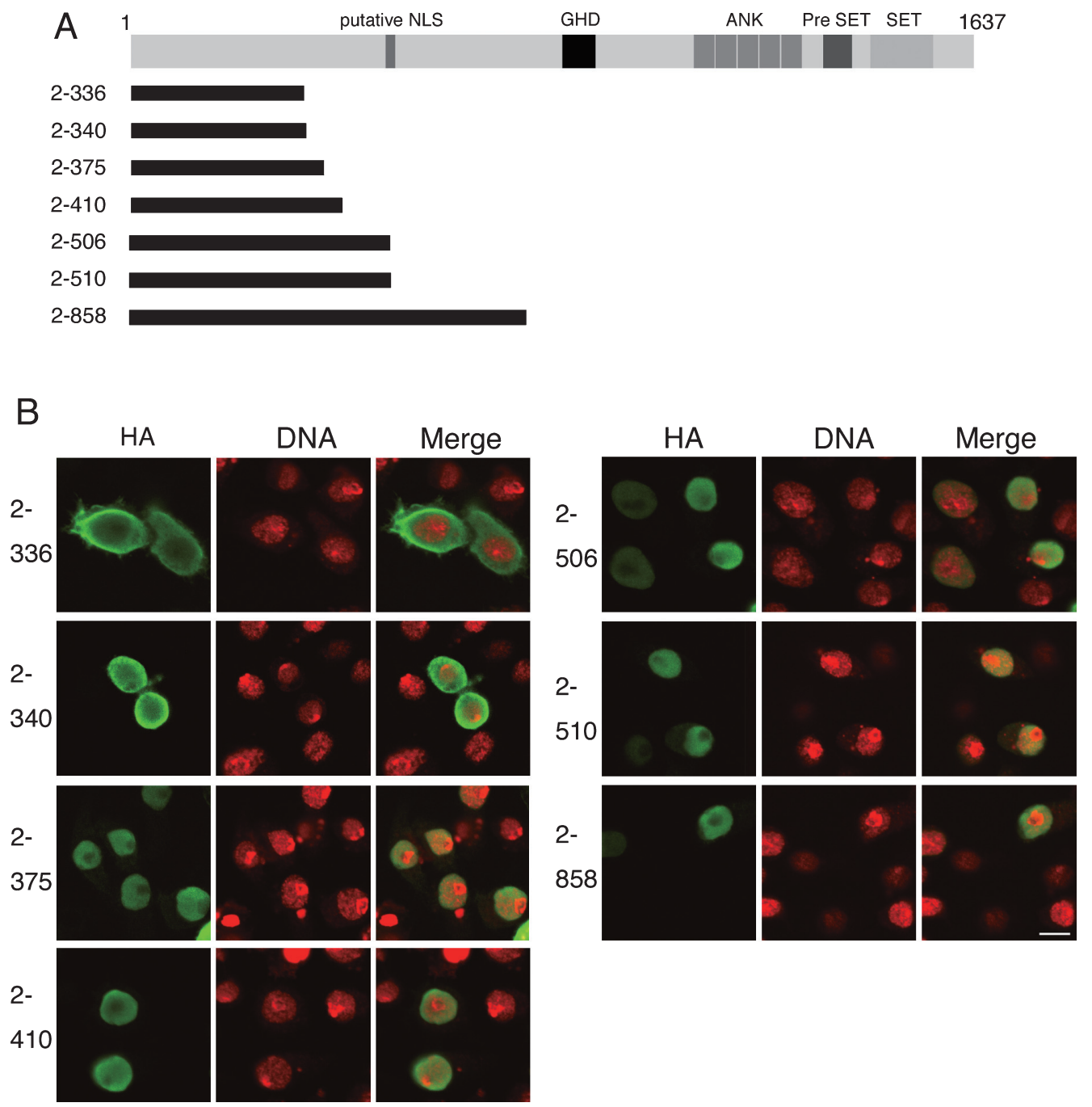

Fig. 2. Examination of the role of the N-terminal region of dG9a in the nuclear localization. (A) A schematic diagram of dG9a wild-type and its Nterminal deletion derivatives used to determine the subcellular localization of dG9a. (B) Confocal laser scanning microscopy of Kc cells transfected with HA-tagged proteins (green). DNA was stained with PI (red). Scale bar, $5 \mu \mathrm{m}$.

$25^{\circ} \mathrm{C}$ in M3 medium (Sigma, St Louis, MO, USA) supplemented with $2 \%$ fetal calf serum in the presence of $5 \% \mathrm{CO}_{2}$ atmosphere. Cells were transfected with pAct5C-GAL4 as the effector plasmid and pUAST constructs or pActSV-HA constructs using CELLFECTIN reagent (Invitrogen, Carlsbad, CA, USA).

\section{Results and Discussion}

\section{Subcellular localization of full-length dG9a and its deletion mutants in Kc cells}

Drosophila G9a localizes in nuclei in salivary glands (Kato et al., 2008) and cultured Drosophila Kc cells (Fig. 1B). The observed rather weaker dG9a signal in propidium iodide (PI)-dense region may represent the localization of dG9a in euchromatic region rather than heterochromatic region in the nucleus as reported previously (Kato et al., 2008). Mammalian G9a has nuclear localization signals (NLS) in its N-terminal region (Estève et al., 2005). Although some domains of G9a such as GHD, ANK, preSET, and SET are conserved between Drosophila and mammals, N-terminal regions including NLS is not highly conserved (Fig. 1A). Amino acid sequences of dG9a in the region from amino acids (aa) 592 to aa651 corresponding to the NLS of mammalian G9a shows only a little conservation (Fig. 1A). The full-length FLAG-tagged dG9a (aa2aa1637) exclusively localized in nuclei as in the case of endogenous dG9a (Fig. 1B). However the truncated FLAGtagged dG9a (aa547-aa809) including the aa592-aa651 
A

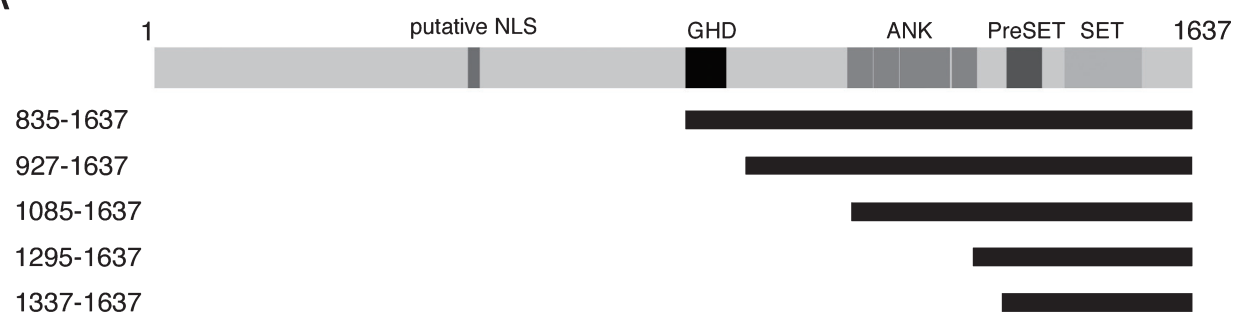

B

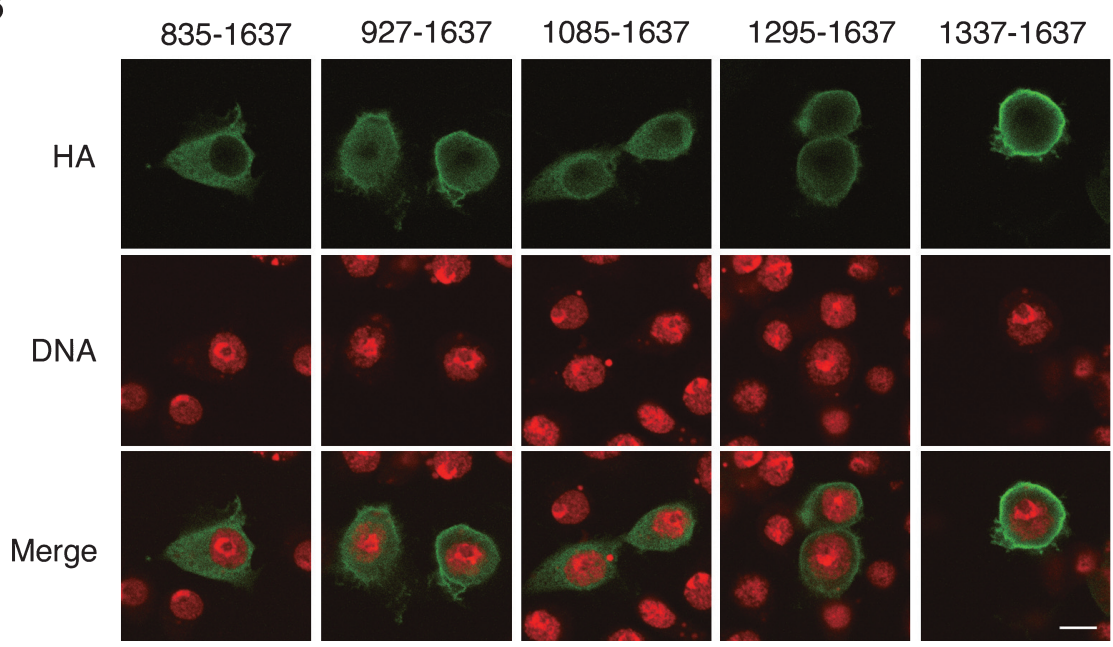

Fig. 3. Examination of the role of the C-terminal region of dG9a in the nuclear localization. (A) A schematic diagram of dG9a wild-type and its Cterminal deletion derivatives used to determine the subcellular localozation of dG9a. (B) Confocal laser scanning microscopy of Kc cells transfected with HA-tagged proteins (green). DNA was stained with PI (red). Scale bar, $5 \mu \mathrm{m}$.

region (Fig. 1C) was mainly localized in the cytoplasm (Fig. 1D). The data indicate that the region corresponding to the NLS of mammalian G9a is not functional as NLS in cultured Drosophila Kc cells.

To determine the region of dG9a required for the nuclear localization, we constructed a set of truncated forms of dG9a mutants (Fig. 1C). Before immunocytochemical analysis, all the truncated mutant constructs were transfected into Drosophila Kc cells, then we prepared the cell extracts and confirmed the expression of mutant proteins as the expected size by Western blotting analysis (data not shown). In the transfected Kc cells, both the full-length dG9a (aa2-aa1637) and the truncated forms of dG9a containing the region aa274-aa546 localized exclusively in nuclei (Fig. 1D). In contrast, the dG9a mutants containing ANK region (aa1085-aa1336) or C-terminal region (aa1337-aa1637) including PreSET and SET domains were exclusively localized in the cytoplasm. In addition, the dG9a containing the aa2-aa273 mainly localized in the cytoplasm (Fig. 1D). These results suggest that the amino acid region aa274-aa546 is responsible for the nuclear localization of dG9a. In this region, a putative NLS (aa506aa512, PKRSKRR) was found by scanning the dG9a protein sequence for NLS motif (KxxKRR) (PredictNLS; http:// cubic.bioc.columbia.edu/predictNLS/) (Stabell et al., 2006). Surprisingly, the amino acid region (aa810-aa1084) containing GHD (aa834-aa926) was also located in nuclei (Fig. 1D, 810-1084). As this domain of mammalian G9a is responsible for the protein interaction (Gyory et al., 2004; Nishida et al., 2007), it may be possible that dG9a interacts with other proteins via this GHD domain to be transported into nuclei.

Next, to determine whether the putative NLS (aa506aa512, PKRSKRR) is responsible for the nuclear localization of dG9a, we constructed an additional set of truncated constructs (Fig. 2A). However, unexpectedly the mutant proteins lacking the putative NLS (aa2-aa375, aa2-aa410, aa2-aa506) were all detected in the nucleus, whereas mutant containing the aa2-aa336 region of dG9a was detected exclusively in the cytoplasm and that containing 
A

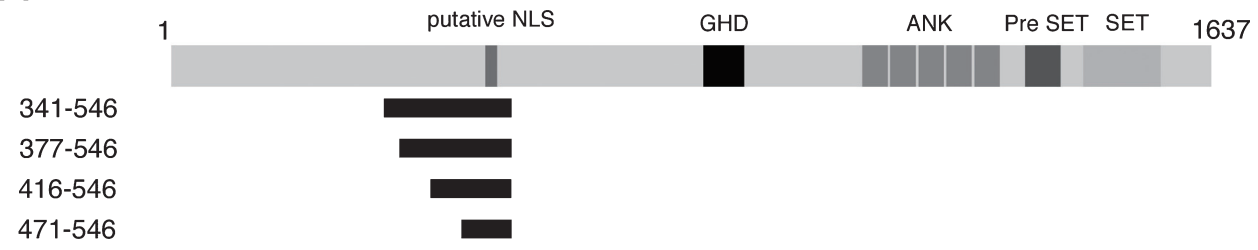

B

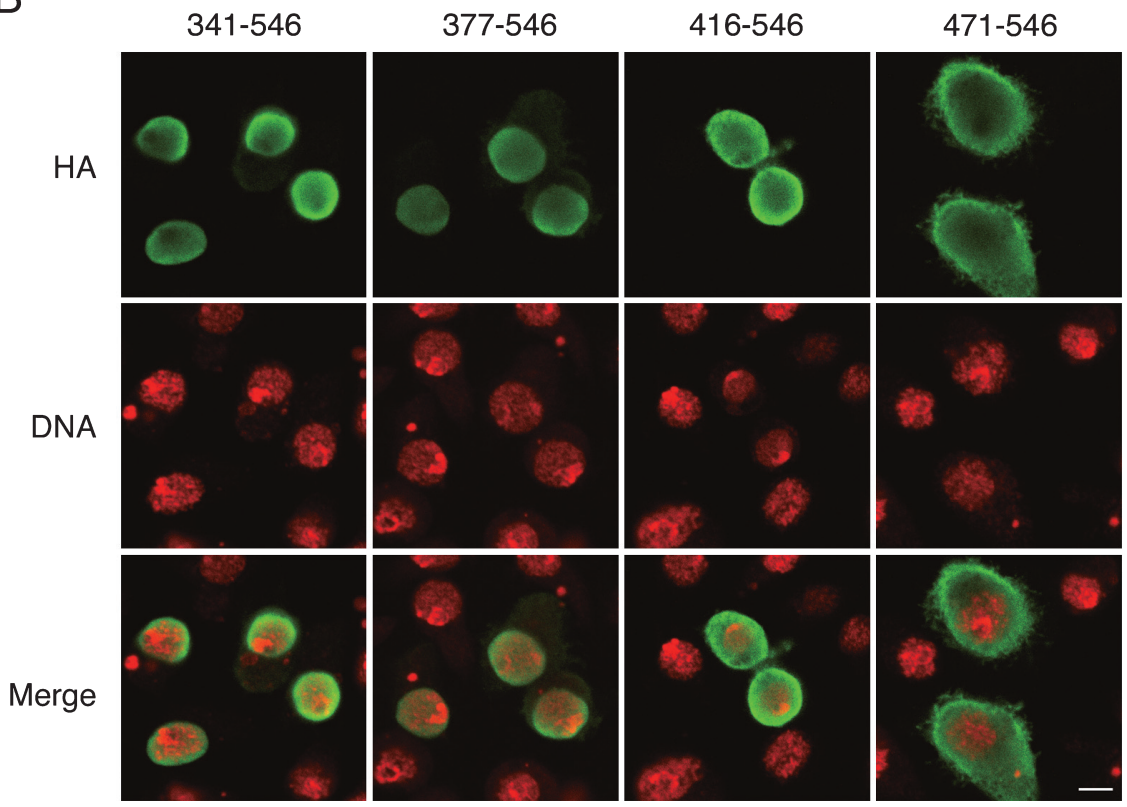

Fig. 4. Dissection of N-terminal region of dG9a required for the nuclear localization. (A) A schematic diagram of dG9a wild-type and its mutants used to determine the subcellular localozation of dG9a. (B) Immunostaining of Kc cells with $\alpha$-HA antibody. Kc cells transfected with HA-tagged proteins (green). DNA was stained with PI (red). Scale bar, $5 \mu \mathrm{m}$.

the aa2-aa340 region was detected diffusively in both nucleus and cytoplasm (Fig. 2B). These results indicate that the predicted NLS (aa506-aa512) is not required for the nuclear localization of $\mathrm{dG} 9 \mathrm{a}$ and the amino acid region aa336-aa375 may be rather responsible for the nuclear localization in cultured $\mathrm{Kc}$ cells.

As the truncated dG9a (aa810-aa1084) containing GHD (aa834-aa926) localized in nucleus (Fig. 1D), we also examined whether the C-terminal region of dG9a is required for nuclear localization (Fig. 3). Although mutant proteins containing aa927-aa1637 and aa1085-aa1637 were detected diffusely in both the cytoplasm and the nucleus, the protein aa835-aa1637 containing GHD did not localize in the nucleus at all (Fig. 3B). Therefore the GHD that is responsible for the protein-protein interaction may not play a role for the nuclear localization of dG9a. The data also suggest that the sequence from aa810 to aa 835 might play a role for the nuclear localization, although the sequence contains neither predictable NLS sequence nor basic lysine and arginine-rich region.
In addition to the above mentioned predictable but not functional NLS-like sequence (aa506-aa512, PKRSKRR), the amino acid region aa274-aa546 of dG9a contains four short stretches of amino acids (aa334-aa345, aa366-aa378, aa407-aa419 and aa461-aa472) that are rich in the basic lysine and arginine residues that may function as NLS motifs (Nigg, 1997; Jans et al., 2000). We therefore examined subcellular localization of truncated forms of dG9a spanning these regions (Fig. 4A). Although the mutant proteins containing aa341-aa546 or aa377-aa546 exclusively localized in the nucleus, that containing aa416-aa546 localized diffusely in both the cytoplasm and the nucleus and that containing aa471-aa546 region exclusively localized in the cytoplasm (Fig. 4B). Taking account of the observation that the mutant protein aa2-aa336 exclusively localizes in the cytoplasm (Fig. 2B), the results suggest that the amino acid sequences responsible for the NLS exist in the region aa337-aa470. 
A
NLS1 330 ENSAPAPKRGRGRAKIRPD 349
NLS2 362 EDSLGEKKPGRKRKLPDEPID 382
NLS3 403 PLGDVKRMRRSVRLGNRLHAD 423
NLS4 457 VLDEKTPPKKRGRKAKTPCV 476

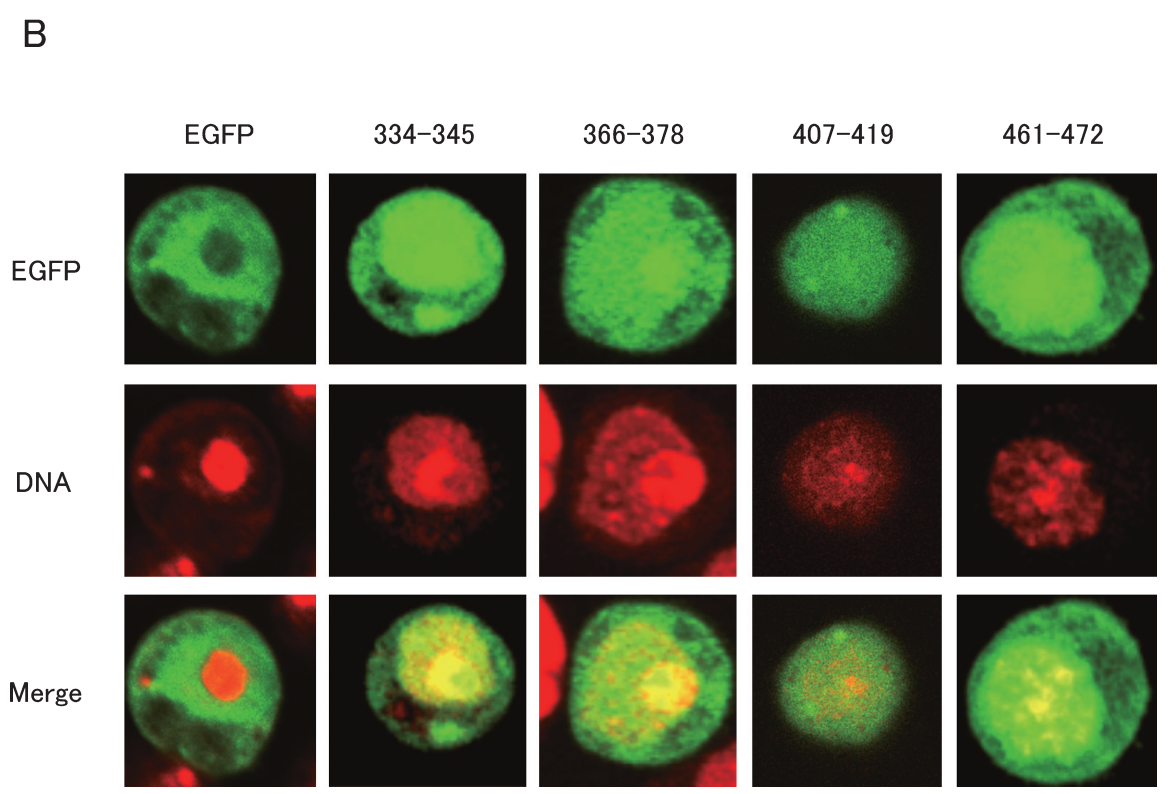

Fig. 5. Detailed mapping of the NLS in dG9a. (A) Candidate NLS sites within amino acids sequence 337-472 in dG9a. (B) Subcellular localization of EGFP and a set of EGFP-tagged putative NLS sequences in Kc cells. Kc cells transfected with EGFP alone or EGFP-tagged proteins (green). DNA was stained with PI (red).

\section{Identification of NLS motifs in dG9a}

Although no potential NLS motif (WoLF PSORT; http:// wolfpsort.org/) was found in the region aa337-aa470, we found four short stretches of positively-charged basic amino acids (NLS1, aa334-aa345; NLS2, aa366-aa378; NLS3, aa407-aa419; NLS4, aa461-aa472) (Fig. 5A). In order to examine the role of the each of these potential NLS motifs within amino acids 337-470, we introduced expression plasmids in which each of these putative NLS was fused with the enhanced green fluorescent protein (EGFP) into Kc cells. Because of the lack of NLS, the expressed EGFP alone exclusively localized in cytoplasm (Fig. 5B). In contrast the EGFP fused with the NLS3 (aa407-aa419) exclusively localized in nuclei and no EGFP signal was detectable in cytoplasm (Fig. 5B). Both EGFPs fused with either the NLS1 (aa334-aa345) or the NLS4 (aa461-aa472) mainly localized in the nucleus with much weaker signals in the cytoplasm. Although the EGFP fused with the NLS2 (aa366-aa378) localized in the nucleus, significant level of the signal was also detected in the cytoplasm. These results indicate that each of the NLS 1, 3 and 4 is sufficient for the nuclear localization with the strongest effect with the NLS3.

To further evaluate roles of the regions aa334-aa472 including NLS1 to 4 and aa834-aa926 including GHD, we have constructed the expression plasmids for dG9a carrying internal deletions in either one or both of these regions (Fig. 6A). dG9a carrying an internal deletion in either one of these regions localized in nuclei (Fig. 6B). However, dG9a carrying internal deletions in both of these regions localized in the cytoplasm (Fig. 6B). The data indicate that the region aa810-aa833 is not sufficient for the nuclear localization. The data taken together with the other data suggest that the region aa810-aa926 in addition to the region aa334-aa472 may be necessary for the nuclear localization. 


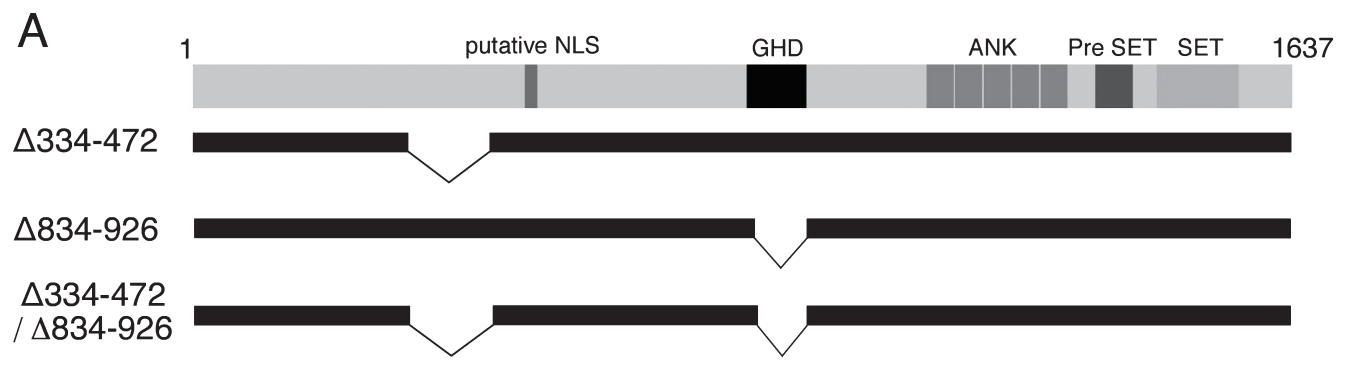

B

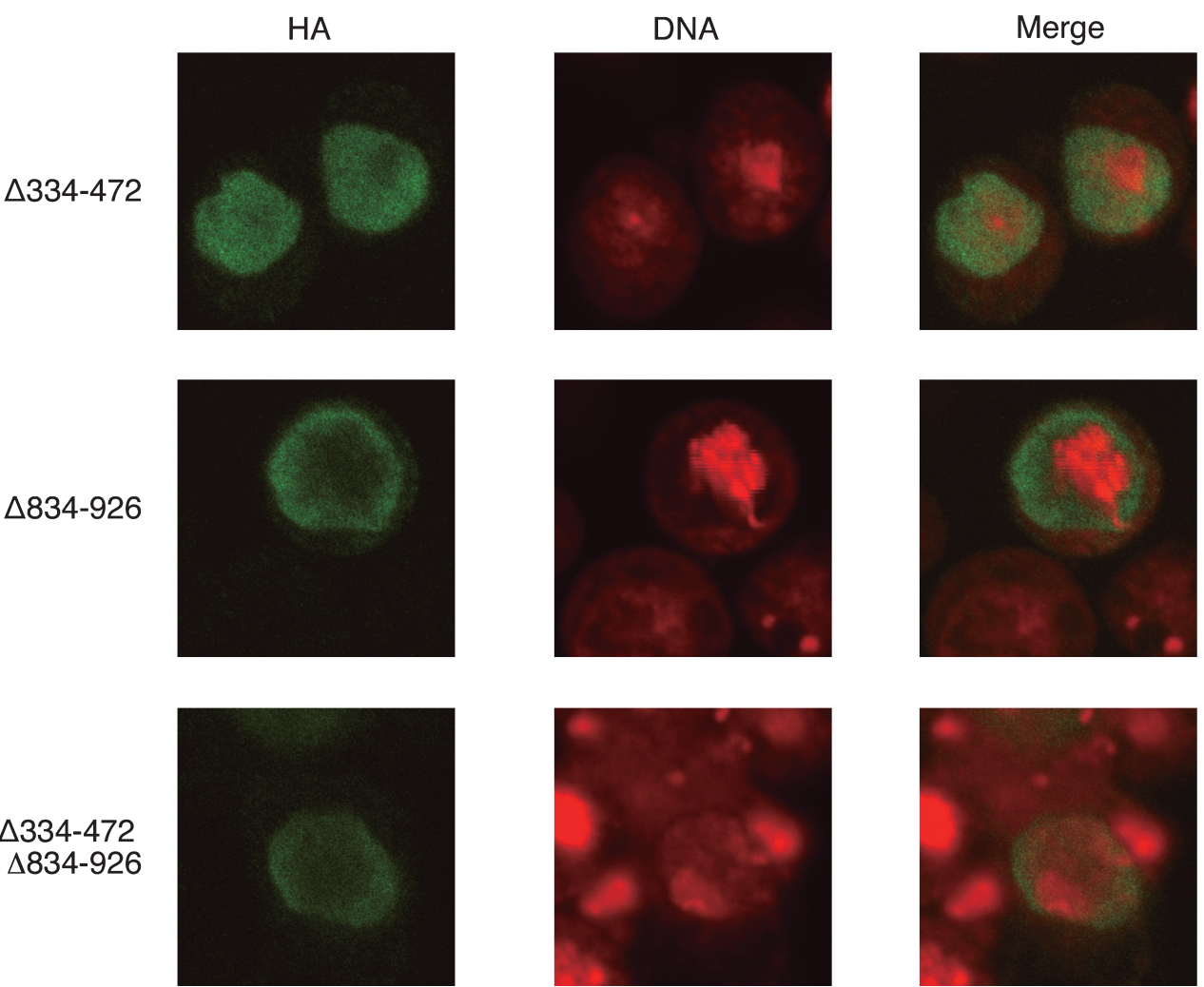

Fig. 6. Subcellular localization of dG9a carrying internal deletions. (A) A schematic diagram of dG9a internal deletion derivatives used for the subcellular localization analysis. (B) Confocal laser scanning microscopy of Kc cells transfected with HA-tagged proteins (green). DNA was stained with PI (red).

NLS of many proteins are conserved between mammals and Drosophila. However in the case of G9a, NLS is not conserved between mammals and Drosophila in its position and the amino acid sequence. The mammalian G9a exclusively localize in nuclei in various cells and tissues. Although Drosophila G9a localize in nuclei in salivary gland cells (Kato et al., 2008) and cultured cells as shown in this study, our preliminary data indicate that dG9a also localize in the cytoplasm at some stages of early embryogenesis (data not shown), suggesting some shuttling activity of dG9a during embryogenesis. The NLS of ancestor form of G9a may be therefore diverged during evolution to adopt each regulatory mechanism for subcellular localization of mammalian and Drosophila G9a.

Acknowledgments. This study was supported by Grants-in-Aid from the Ministry of Education, Culture, Sports, Science and Technology of Japan.

\section{References}

Brower-Toland, B., Riddle, N.C., Jiang, H., Huisinga, K.L., and Elgin, S.C.R. 2009. Multiple SET merhyltransefeseases are required to maintain normal heterochromatin domains in the genome of Drosophila melanogaster. Genetics, 181: 1303-1319.

Czermin, B., Shotta, G., Hülsmann, B.B., Brehm, A., Becker, P.B., Reuter, G., and Imhof, A. 2001. Physical and functional association of 
SU(VAR)3-9 and HDAC1 in Drosophila. EMBO Rep., 2: 915-919.

Ebert, A., Schotta, G., Lein, S., Kubicek, S., Krauss, V., Jenuwein, T., and Reuter, G. 2004. Su(var) genes regulate the balance between euchromatin and heterochromatin in Drosophila. Genes Dev., 18: 2973-2983.

Estève, P.O., Patnaik, D., Chin, H.G., Benner, J., Teitell, M.A., and Pradhan, S. 2005. Functional analysis of the N- and C-terminus of mammalian G9a histone H3 methyltransferase. Nucleic Acids Res., 10: 3211-3223.

Fritsch, L., Robin, P., Mathieu, J.R., Souidi, M., Hinaux, H., Rougeulle, C., Harel-Bellan, A., Ameyar-Zazoua, M., and Ait-Si-Ali, S. 2010. A subset of the histone H3 lysine 9 methyltransferases Suv39h1, G9a, GLP, and SETDB1 participate in a multimeric complex. Mol. Cell, 37: 46-56.

Gyory, I., Wu, J., Fejér, G., Seto, E., and Wright, K.L. 2004. PRDI-BF1 recruits the histone $\mathrm{H} 3$ methyltransferase G9a in transcriptional silencing. Nat. Immunol., 5: 299-308.

Jans, D.A, Xiao, C.Y., and Lam, M.H. 2000. Nuclear targeting signal recognition: a key control point in nuclear transport? BioEssays, 22: $532-544$.

Kato, Y., Kato, M., Tachibana, M., Shinkai, Y., and Yamaguchi, M. 2008. Characterization of Drosophila G9a in vivo and identification of genetic interactants. Genes Cells, 181: 1303-1319.

Lee, K.-S., Yoon, J., Park, J.S., and Kang, Y.-K. 2010. Drosophila G9a is implicated in germ cell development. Insect Mol. Biol., 19: 131-139.

Mang, L., Charroux, B., Kerridge, S., and Tsai, C.C. 2008. Atrophin recruits $\mathrm{HDAC} 1 / 2$ and $\mathrm{G} 9 \mathrm{a}$ to modify histone $\mathrm{H} 3 \mathrm{~K} 9$ and to determine to cell fate. EMBO Rep., 9: 555-562.

Martin, C. and Zhang, Y. 2005. The diverse functions of histone lysine methylation. Nat. Rev. Mol. Cell Biol., 6: 838-849.

Mis, J., Ner, S.S., and Grigliatti, T.A. 2006. Identification of three histone methyltrasnferases in Drosophila: dG9a is a suppressor of PEV and is required for gene silencing. Mol. Genet. Genomics, 275: 513-526.

Nigg, E.A. 1997. Nucleocytoplasmic transport: signals, mechanisms and regulation. Nature, 386: 779-787.
Nishida, M., Kato, M., Kato, Y., Ueda, J., Tachibana, M., Shinkai, Y., and Yamaguchi, M. 2007. Identification of ZNF200 as a novel binding partner of histone H3 methyltransferase G9a. Genes Cells, 12: 877-888.

Schotta, G., Ebert, A., Krauss, V., Fischer, A., Hoffmann, J., Rea, S., Jenuwein, T., Dorn, R., and Reuter, G. 2002. Central role of Drosophila SU(VAR)3-9 in histone H3-K9 methylation and heterochromatic gene silencing. EMBO J., 21: 1121-1131.

Seum, C., Reo, E., Peng, H., Rauscher, F.J., 3rd, Spierer, P., and Bontron, S. 2007. Drosophila SETDB1 is required for chromosome 4 silencing. PLoS Genet., 3: e76.

Stabell, M., Eskeland, R., Bjørkmo, M., Larsson, J., Aalen, R.B., Imhof, A., and Lambertsson, A. 2006. The Drosophila G9a gene encodes a multiple-catalytic histone methyltransferase required for normal development. Nucleic Acids Res., 34: 4609-4621.

Sugano, W. and Yamaguchi, M. 2008. Identification of novel localization signals of Drosophila myeloid leukemia factor. Cell Struct. Funct., 32: 163-169.

Tachibana, M., Ueda, J., Fukuda, M., Takeda, N., Ohta, T., Iwanari, H., Sakihama, T., Kodama, T., Hanakubo, T., and Shinkai, Y. 2005. Histone methyltransferases G9a and GLP form heteromeric complexes and are both crucial for methylation of euchromatin at H3-K9. Genes Dev., 19: 815-826.

Tzeng, T.Y., Lee, C.H., Chan, L.W., and Shen, C.K. 2007. Epigenetic regulation of the Drosophila chromosom 4 by the histone H3K9 methyltransferase dSETDB1. Proc. Natl. Acad. Sci. USA, 104: 12691-12696.

Yokochi, T., Poduch, K., Ryba, T., Lu, J., Hiratani, I., Tachibana, M., Shinkai, Y., and Gilbert, D.M. 2009. G9a selectively represses a class of late-replicating genes at the nuclear periphery. Proc. Natl. Acad. Sci. USA, 106: 19363-19368.

(Received for publication, December 24, 2010, accepted, April 16, 2011 and published online, April 22, 2011) 